\title{
Reimbursement for Continuous Glucose Monitoring
}

\author{
Lutz Heinemann, PhD, and J. Hans DeVries, MD, PhD²
}

\begin{abstract}
Continuous glucose monitoring (CGM) systems have been available for more than 15 years by now. However, market uptake is relatively low in most countries; in other words, relatively few patients with diabetes use CGM systems regularly. One major reason for the reluctance of patients to use CGM systems is the costs associated (i.e., in most countries no reimbursement is provided by the health insurance companies). In case reimbursement is in place, like in the United States, only certain patient groups get reimbursement that fulfills strict indications. This situation is somewhat surprising in view of the mounting evidence for benefits of CGM usage from clinical trials: most meta-analyses of these trials consistently show a clinically relevant improvement of glucose control associated with a reduction in hypoglycemic events. More recent trials with CGM systems with an improved CGM technology showed even more impressive benefits, especially if CGM systems are used in different combinations with an insulin pump (e.g., with automated bolus calculators and low glucose suspend features). Nevertheless, sufficient evidence is not available for all patient groups, and more data on costefficacy are needed. In addition, good data from real-world studies/registers documenting the benefits of CGM usage under daily life conditions would be of help to convince healthcare systems to cover the costs of CGM systems. In view of the ongoing improvements in established needle-type CGM systems, the fact that new CGM technology will come to the market soon (e.g., implantable sensors), that CGM-like systems are quite successfully at least in certain markets (like the flash glucose monitoring systems), and that the first artificial pancreas systems will come to the market in the next few years, there is a need to make sure that this major improvement in diabetes therapy becomes more widely available for patients with diabetes, for which better reimbursement is essential.
\end{abstract}

\section{Introduction}

A FTER THE APPROVAL OF THE FIRST SYSTEMS for continuous glucose monitoring (CGM) some 15 years ago, many expected a "quantum leap" in diabetes therapy. The hope was that CGM would be supportive in reducing the daily variations in glycemia in people with diabetes and thereby prevent both hypoglycemia and hyperglycemia. In this sense, CGM does not simply represent a different type of self-monitoring of blood glucose (SMBG); this technique opens up totally new perspectives for the self-management of diabetes and paves the way for an artificial pancreas (AP) system. Starting from these considerations, one would have expected CGM systems to have become standard diabetes therapy by now, with full reimbursement by health insurance systems. This is certainly not the case, and it is important to discuss the reasons for the slow acceptance.

One of the reasons is technical shortcomings. Even with the most recent CGM systems,
- The glucose sensors have to be replaced after a few days.

- The sensor has to be inserted through the skin into the subcutaneous adipose tissue.

- Accuracy of the sensor measurement is not always optimal.

- There is still need for initial calibration with a SMBG sample and for more or less frequent recalibrations.

We have seen a number of generations of CGM systems coming to the market, each bringing improvements in analytical performance and handling, but CGM usage has not become "viral" (i.e., only a minority of patients uses CGM systems regularly, with considerable differences between countries, also depending on the respective reimbursement situation). For example, there are interesting differences in the reimbursement policy for CGM across Europe. ${ }^{1}$

It is obvious that CGM provides much more information to patients with diabetes than self-measurement of capillary

\footnotetext{
${ }^{1}$ Science \& Co., Düsseldorf, Germany.

${ }^{2}$ Academic Medical Center at the University of Amsterdam, Amsterdam, The Netherlands.
} 
blood glucose, but it turns out that in practice many patients use this additional information in a way that enables them to reduce the risks of diabetes therapy ( $=$ avoid acute metabolic deteriorations), but not necessarily to systematically optimize glycemic control resulting in an improved glycated hemoglobin (HbA1c) level. Most of us know patients who can testify to the massive change/help that CGM represents for them in daily life. Sometimes such patients are willing to pay out of pocket for this option; for them the benefits of CGM are so large that they are prioritizing this over spending on other items. Talking to such patients and seeing the improvements in their glycemic profiles make one wonder why results of clinical trials with CGM are not equally overwhelming. ${ }^{2}$

The aim of this article is to discuss aspects that are relevant for reimbursement of CGM and ways to improve the situation.

\section{Cost of CGM}

Assuming that the daily costs for CGM usage are of the order of $\$ 5-10$ per day, this amounts to $\$ 3,000$ per year per patient. In some European countries the cost is around $4,000 €$ per year. Being conservative and assuming also that there might be 1 million potential users with type 1 diabetes in the United States, this would total approximately 3 billion dollars each year. This number makes it clear right away why health insurance companies are so reluctant to promote widespread use of such a cost driver with-from their point of view-no clear or unique benefit that necessitates such an investment: CGM is not a lifesaving intervention. Many patients with diabetes survive relatively well either with SMBG alone or sometimes even without any regular monitoring of their daily blood glucose excursions whatsoever. On the other hand, huge proportions of patients with diabetes do not achieve satisfactory glycemic control, which enhances the risk of developing diabetes-related late complications and their associated cost. The use of CGM might be of help in this respect.

The considerable costs of CGM systems are claimed by the manufacturers to be due not only to the huge investment in the lengthy development and approval process of CGM systems, but also to the elaborate production process of the systems themselves (in particular, the actual sensors), a substantial part of which is still performed manually. Automated production would result in a significant reduction in the cost per sensor. Perhaps other innovative CGM systems will come to the market in the future with improved accuracy and manufacturing processes that will lower the costs of CGM systems. Of note is that the recently introduced Abbott (Alameda, CA) FreeStyle ${ }^{\circledR}$ Libre system, further discussed below, was brought to the market at a price per day of onethird to one-half that of other current systems, as it can be used for 14 days rather than 5 days with the former Abbott system.

Use of CGM requires time by healthcare professionals to train patients as well as to see the patients for repeated visits to the diabetes outpatient centers for interpretation of the CGM profiles. The costs of this time must be added to the cost of CGM systems.

\section{CGM: Dynamic Technology Development}

A big advantage and a disadvantage at the same time are that CGM technology is developing quite rapidly. As mentioned, each and every year in the last 15 years new genera- tions of CGM systems with improved properties have come to the market. This is good for the patients but hampers documentation of evidence for CGM usage. Performance of clinical studies with a given CGM system with a sufficient study duration and sample size lasts easily $2-3$ years. By the time the study data are published the CGM system studied is outdated.

The rapid improvement in CGM technology also hampers comparability of results of study findings obtained over time (making meta-analysis a challenge): the experimental conditions under which the studies were performed might have remained more or less comparable; however, for example, the analytical performance (= quality with which glucose can be measured, especially in the low glucose range) and many other features were quite different in studies performed some 10 years ago versus studies with recent CGM systems. Nevertheless, the mean absolute relative difference reported for the originally approved CGM devices was in the range of $25 \%$, is $<15 \%$ for most of the current versions, and is $10 \%$ for the most recent versions and those that will come to the market in the next few years. Thus, making statements about the clinical value of CGM systems based on older studies can be misleading (e.g., the increased rate of severe hypoglycemic events seen in an early study like STAR1 can likely in part be explained by the fact that it was performed with technology now outdated ${ }^{3}$ ). Applying a pragmatic approach and include only studies from the last 4 or 5 years in metaanalysis is hampered by the fact that no CGM studies with all relevant patient groups were performed in these years (see below). Additionally, the more recent CGM generations are much more user friendly and easier to use than the first generations: these were simply too large, and insertion of the needle was too painful for kids. This improved user friendliness is likely to translate into more intensive usage, which is associated with lower HbA1c values. ${ }^{4}$

CGM per se is a diagnostic tool (i.e., it does not induce any therapeutic intervention on its own). As long as the patients are involved in making therapeutic decisions, it is key that they are very well trained when starting with this technology; moreover, they should also be continuously supported while using the system. The importance of such psychosocial factors and adequate teaching programs was somewhat ignored in the first years of CGM usage. In the CGM studies published so far such teaching and training aspects were most often not presented at all or only quite briefly. As to be expected there was also a clear learning curve of investigators and patients in the usage of CGM.

\section{What Are We Talking About? CGM Per Se or in Combination with Continuous Subcutaneous Insulin Infusion/Sensor-Augmented Pump/Low Glucose Suspend/Predictive Low Glucose Management/AP}

In most of the recent studies the CGM system was not studied per se but in combination with an insulin pump. These combinations, which are obvious steps toward an AP, bring together the best diagnostic technology with the best therapeutic option. However, only a minority of patients with type 1 diabetes uses continuous subcutaneous insulin infusion therapy. There is a lack of knowledge about the value of CGM in patients on multiple daily insulin injections. Thus, from the perspective of somebody who has the job to evaluate 
the clinical evidence of CGM and make a decision about reimbursement, it is hard to justify reimbursement for the majority of patients (i.e., those on injection therapy).

In case the patients use a combination of a CGM system with a pump, not the benefit of CGM per se was studied, but the combined effect of two interventions (if the patients were not using a pump before). Is the achieved decline in $\mathrm{HbAlc} /$ reduction in hypoglycemia frequency due to the beneficial effect of the CGM system or the insulin pump? And how to disentangle the contribution of the bolus advisor or other properties built into modern pumps? From a clinical point of view this may not really matter in the end, but this is different for reimbursement people, especially in view of the considerable costs of both technological systems combined.

\section{Evidence for CGM Usage as Documented by Meta-Analysis}

In the last years several reviews and also guidelines about CGM were published. ${ }^{5}$ There are certain differences in the outcome of these meta-analyses; these are mainly driven by the different approaches taken by the authors, resulting in a different set of clinical studies included and a different way of interpreting the evidence. In other words, depending on the set of inclusion/exclusion criteria used, as well as the criteria for pooling, the results obtained are quite different. In our opinion this also reflects several shortcomings of CGM studies performed so far (see below).

A methodologically superior approach was taken by Pickup et al. "They did not "simply" combine the mean values of a given set of parameters reported in the respective studies, but were able to obtain individual patient data from a set of clinical studies sponsored by one company. This analysis showed benefits of using CGM for HbAlc and hypoglycemic events, especially when using the CGM device regularly, and represents the best proof for the benefits of CGM from our point of view.

One of us (J.H.D.) was involved in a Cochrane review of $\mathrm{CGM}^{2}$ Analyzing trials in such a formalized way (while not forgetting the clinical usage of CGM) was helpful in seeing the shortcomings of CGM studies. Many of these studies (except, for example, the JDRF study) were performed by or in close cooperation with the manufacturer of the CGM system studied. The number of patients included was sufficient from a marketing and registration point of view, but the data were often insufficient to allow critical evaluation in order to be convincing for reimbursement authorities. One can argue that one of the main reasons why the evidence for CGM is not overwhelming from the older studies is that in most studies patients most likely to benefit were not included (i.e., those with frequent severe hypoglycemia or persistently elevated $\mathrm{HbA} 1 \mathrm{c}$ levels on conventional treatment). If there is no problem with hypoglycemia or HbA1c to start with, CGM cannot improve it.

As outlined above, just by adding studies with newer CGM systems to such meta-analysis will make them more convincing. You cannot "heal" studies with an inappropriate study design, etc., by combining several such studies in one larger analysis. We see the need for "fresh" and good data, acknowledging at the same time that most manufacturers of CGM systems are not willing to perform such studies over and over again with each new CGM generation (plus the movement to combinations with pumps/AP systems). How- ever, from a reimbursement point of view we still see a need for a relatively small number of larger studies with a sufficient study duration, selection of the right patients groups, etc., to document evidence of CGM usage in all patient groups/indications. It is worth mentioning that there is the risk that the outcome might be negative in certain patient groups (i.e., in those patients who make use of CGM for their own purposes/interests, which might be different from that of healthcare providers)!

Recently the IQWiG in Germany published their evaluation of the benefits of CGM usage. ${ }^{6,7}$ The positive outcome of this analysis (at least for certain patient groups) will most probably lead to reimbursement for CGM becoming available in Germany, at least for certain patient groups/ indications. Also, NICE recently published clinical guidelines that recommend CGM in certain adults and children with type 1 diabetes, centering on those patients with frequent unexplained severe hypoglycemic events and hypoglycemia unawareness (https://www.diabetes.org.uk/Global/Homepage/ News/NG17\%20Guidance\%20Type\%201\%20diabetes \%20 in\%20adults.pdf). One of the recommendations seems not clearly formulated where asymptomatic hypoglycemia is mentioned as a criterion, if this affects daily activities. It is unclear how it could be assessed whether asymptomatic hypoglycemia affects daily activities. In addition, patients must be willing to use CGM at least $70 \%$ of the time, and CGM should only be continued if the HbA1c level can be sustained at $<7 \%$ and/or there has been a reduction in HbA1c level of $>2.5 \%$. So, in two major European markets, at least for certain patient groups, reimbursement for CGM is likely to become available.

\section{Evidence from Larger Recent Studies}

In our opinion the JDRF CGM study is one of the best studies available; not only was this sponsored by an independent organization and not by one of the manufacturers, it also included a considerable number of patients and had a relatively long study duration. ${ }^{8}$ It was published in a prestigious journal and represents one of the very few "class studies." In this study three different CGM systems were used, but the study data were not analyzed separately for each of the CGM devices (at least such data were never published). The aim of the study was to evaluate the benefit of CGM per se on glycemic control in three different patient groups (separated by age). No benefit was seen in the younger age groups, as the kids and teens used the CGM systems less than the adults, for whom a benefit was seen. Apparently the technology was not at the level expected by these age groups, where especially the adolescents are generally less motivated to engage actively with the disease. As technology has improved in the last years (see above) the outcome of more recent studies was more positive, also in the younger age groups. ${ }^{9}$

STAR3 and the Eurythmics study confirmed that the sensor pump combination with bolus calculator (sensor-augmented pum therapy) is superior to standard injection therapy. ${ }^{10,11}$ ASPIRE confirmed that sensor-augmented pump/low glucose suspend diminishes time in hypoglycemia. ${ }^{10,12,13}$

Whereas these studies did not report a reduction in severe hypoglycemic events, a recently reported study from Australia did so, focusing on patients with hypoglycemia unawareness. ${ }^{14}$ This randomized controlled trial confirmed the 
results of a recent observational study in people with hypoglycemia unawareness. ${ }^{15}$ However, the study results were questioned by the IQWiG in their CGM report based on the statistical analysis method used. ${ }^{16}$

\section{Cost-Benefit of CGM Systems}

In cost-benefit analyses of CGM systems, the authors use health economic models and assumptions to investigate whether the use of CGM systems optimizes glycemic control to such an extent that the reduction in the cost of treating diabetes-related complications outweighs the cost of using the CGM systems or even makes their use cost saving. Usually, quality-adjusted life years gained and the corresponding incremental cost-effectiveness ratio are calculated to provide evidence for cost-effectiveness. The only cost-efficacy analysis published so far showed an unfavorable cost-efficacy ratio; however, a new analysis in the subgroups of patients with severe hypoglycemia and substantial lowering of HbAlc hopefully will show a more acceptable cost-efficacy. ${ }^{17}$ This is important as reimbursement is largely decided on costeffectiveness. The thought here is that it might be that an expensive technology effectively reduces HbAlc and hypoglycemia, but if the incremental cost-effectiveness ratio is above the "willingness-to-pay" threshold for that country/ healthcare scheme, it will not be reimbursed.

\section{Recent Experience with a CGM-Like System in Germany}

At the end of 2014 a novel glucose monitoring system (the FreeStyle Libre by Abbott) was introduced in the German market. The experience is that thousands of patients were rapidly switching to this system (also measuring glucose in interstitial fluid), probably also triggered by the quite positive media uptake. Many of these patients are patients with type 2 diabetes who have to date performed capillary blood glucose measurement. Most probably even more patients would have started to use this system if the manufacturer had not struggled with limited production capacities.

As noted above, the costs per day for this system are approximately 50-70\% lower than that of CGM. It appears as if the patients see so many benefits with this approach (=no need for finger pricking at all as this system requires no calibration) that they are willing to pay these costs out of pocket. As of today at least some health insurance companies in Germany are willing to cover the costs for this system despite the fact that the evidence from clinical studies supporting this system (as they always ask for CGM system) is virtually absent, although such studies are currently being performed.

\section{Summary and Outlook}

We see a clear need to have an open discussion about which level of clinical evidence we have for CGM and what are ways to improve, as well as to make access to CGM easier through reimbursement for selected patient groups. We also see shortcomings in the studies performed so far, especially when looking at them with a more methodological/ reimbursement point of view and not so much with a clinical view. However, one has to accept the careful view of the insurance companies on such developments/data: the potential costs they see for our healthcare systems are tremendous.
If we believe in the role/position of CGM, it is our task to provide good evidence for using this technology.

There seems a reasonable justification for reimbursement for patients in poor control who are able to considerably lower their HbA1c level using the device, as well as for people with hypoglycemia unawareness who have encountered severe hypoglycemia. Reimbursement authorities might ask for an even larger trial in selected patients with hypoglycemia unawareness.

In summary, although reimbursement authorities always like to see more evidence, we believe that the recently published studies provide important additional evidence for usage and reimbursement of CGM.

\section{Author Disclosure Statement}

L.H. advises various companies in the development of new diagnostic and therapeutic approaches to diabetes therapy. $\mathrm{He}$ is a shareholder and consultant at Profil Institute for Metabolic Research, Neuss and Profil Institute for Clinical Research, San Diego, CA. J.H.D. is an advisory board member for Roche Diagnostics and has received research support from Abbott, Dexcom, Medtronic, and Senseonics.

\section{References}

1. Heinemann L, Franc S, Phillip M, Battelino T, AmpudiaBlasco FJ, Bolinder J, Diem P, Pickup J, DeVries JH: Reimbursement for continuous glucose monitoring: a European view. J Diabetes Sci Technol 2012;6:1498-1502.

2. Langendam MW, Luijf YM, Hooft L, DeVries JH, Mudde $\mathrm{AH}$, Scholten RJ: Continuous glucose monitoring systems for type 1 diabetes mellitus. Cochrane Database Syst Rev 2012;1:CD008101.

3. Hirsch IB, Abelseth J, Bode BW, Fischer JS, Kaufman FR, Mastrototaro J, Parkin CG, Wolpert HA, Buckingham BA: Sensor-augmented insulin pump therapy: results of the first randomized treat-to-target study. Diabetes Technol Ther 2008;10:377-383.

4. Pickup JC, Freeman SC, Sutton AJ: Glycaemic control in type 1 diabetes during real time continuous glucose monitoring compared with self monitoring of blood glucose: meta-analysis of randomised controlled trials using individual patient data. BMJ 2011;343:d3805.

5. Klonoff DC, Buckingham B, Christiansen JS, Montori VM, Tamborlane W, Vigersky R, Wolpert H: Continuous glucose monitoring: an Endocrine Society clinical practice guideline. J Clin Endocrinol Metab 2011;96:2968-2979.

6. IQWiG: Continuous Glucose Monitoring (CGM) with Real-Time Measurement Devices Has Added Benefit. May 21, 2015. https://www.iqwig.de/en/press/press-releases/pressreleases/continuous-glucose-monitoring-cgm-with-real-timemeasurement-devices-has-added-benefit.6711.html (accessed October 7, 2015).

7. IQWiG: Continuous Interstitial Glucose Monitoring (CGM) with Real-Time Measurement Devices in Insulin-Dependent Diabetes Mellitus. Executive Summary. March 25, 2015. https:// www.iqwig.de/download/D12-01_Executive-Summary_ Continuous-glucose-monitoring-CGM-with-real-time-measure ment-devices.pdf (accessed October 7, 2015).

8. Tamborlane WV, Beck RW, Bode BW, Buckingham B, Chase HP, Clemons R, Fiallo-Scharer R, Fox LA, Gilliam LK, Hirsch IB, Huang ES, Kollman C, Kowalski AJ, Laffel L, Lawrence JM, Lee J, Mauras N, O’Grady M, Ruedy KJ, 
Tansey M, Tsalikian E, Weinzimer S, Wilson DM, Wolpert $\mathrm{H}$, Wysocki T, Xing D: Continuous glucose monitoring and intensive treatment of type 1 diabetes. N Engl J Med 2008; 359:1464-1476.

9. Battelino T, Phillip M, Bratina N, Nimri R, Oskarsson P, Bolinder J: Effect of continuous glucose monitoring on hypoglycemia in type 1 diabetes. Diabetes Care 2011;34: 795-800.

10. Bergenstal RM, Tamborlane WV, Ahmann A, Buse JB, Dailey G, Davis SN, Joyce C, Peoples T, Perkins BA, Welsh JB, Willi SM, Wood MA: Effectiveness of sensoraugmented insulin-pump therapy in type 1 diabetes. N Engl J Med 2010;363:311-320.

11. Hermanides J, Norgaard K, Bruttomesso D, Mathieu C, Frid A, Dayan CM, Diem P, Fermon C, Wentholt IM, Hoekstra JB, DeVries JH: Sensor-augmented pump therapy lowers $\mathrm{HbA}_{1 \mathrm{c}}$ in suboptimally controlled Type 1 diabetes; a randomized controlled trial. Diabet Med 2011;28:11581167.

12. Bergenstal RM, Klonoff DC, Garg SK, Bode BW, Meredith M, Slover RH, Ahmann AJ, Welsh JB, Lee SW, Kaufman FR: Threshold-based insulin-pump interruption for reduction of hypoglycemia. N Engl J Med 2013;369:224-232.

13. Danne T, Kordonouri O, Holder M, Haberland H, Golembowski S, Remus K, Blasig S, Wadien T, Zierow S, hartmann R, Thomas A: Prevention of hypoglycemia by using low glucose suspend function in sensor-augmented pump therapy. Diabetes Technol Ther 2011;13:1129-1134.
14. Ly TT, Nicholas JA, Retterath A, Lim EM, Davis EA, Jones TW: Effect of sensor-augmented insulin pump therapy and automated insulin suspension vs standard insulin pump therapy on hypoglycemia in patients with type 1 diabetes: a randomized clinical trial. JAMA 2013;310:1240-1247.

15. Choudhary P, Ramasamy S, Green L, Gallen G, Pender S, Brackenridge A, Amiel SA, Pickup JC: Real-time continuous glucose monitoring significantly reduces severe hypoglycemia in hypoglycemia-unaware patients with type 1 diabetes. Diabetes Care 2013;36:4160-4162.

16. Heinemann L, Hermanns N: IQWiG reanalyzes and raises questions about an article by Ly et al. which concluded low glucose suspend is very beneficial. J Diabetes Sci Technol 2015 August 6 [Epub ahead of print]. doi: 10.1177/ 1932296815597918.

17. Huang ES, O’Grady M, Basu A, Winn A, John P, Lee J, Meltzer D, Kollman C, Laffel L, Tamborlane W, Weinzimer S, Wysocki T: The cost-effectiveness of continuous glucose monitoring in type 1 diabetes. Diabetes Care 2010;33:12691274.

Address correspondence to: Lutz Heinemann, PhD Science \& Co. Kehler Strasse 24 40468 Düsseldorf, Germany

E-mail:1.heinemann@science-co.com 